\title{
Whole transcriptome data of uninfected and Nosema ceranae-infected midguts of eastern honeybee workers
}

Huazhi Chen", Dingding Zhou\#, Yu Du, Cuiling Xiong, Yanzhen Zheng, Dafu Chen, Rui Guo*

College of Animal Sciences (College of Bee Science), Fujian Agriculture and Forestry University, Fuzhou 350002, China

*Corresponding author: Tel: +86-0591-87640197; Fax: +86-0591-87640197; E-mail address: ruiguo@fafu.edu.cn

\begin{abstract}
Apis cerana cerana is a subspecies of eastern honeybee, Apis cerana. Nosema ceranae is a widespread fungal parasite of honeybee, causing heavy losses for beekeeping industry all over the world. In this article, total RNA of normal midguts (AcCK1, AcCK2) and N. ceranae-infected midguts of A. c. cerana workers at $7 \mathrm{~d}$ and $10 \mathrm{~d}$ post inoculation (AcT1, AcT2) were respectively isolated followed by strand-specific cDNA library construction and next-generation RNA sequencing. In tolal, 56270223688, 44860946964, 78991623806, and 92712308296 raw reads were derived from AcCK1, AcCK2, AcT1 and AcT2, respectively. Following strict quality control, 54495191388, 43570608753, 76708161525, and 89467858351 clean reads were obtained, with Q30 value of 95.80\%, 95.99\%, 96.07\% and 96.04\%, and GC content of $44.20 \%, 43.44 \%, 44.83 \%$ and $43.63 \%$, respectively. The raw data were submitted to the NCBI Sequence Read Archive database and connected to BioProject PRJNA562784. These data offers a valuable resource for deep investigation of mechanisms underlying eastern honeybee responding to $N$. ceranae infection and host-fungal parasite interaction during microsporidiosis .
\end{abstract}

\section{Keywords}


transcriptome, eastern honeybee, Apis cerana cerana, Nosema ceranae,midgut, non-coding RNA

\section{Value of the Data}

- Current dataset offers a valuable resource for exploring mRNAs, lncRNAs and circRNAs involved in response of A. c. cerana worker to N. ceranae infection.

- The accessible data can be used to investigate differential expression pattern and regulatory network of non-coding RNAs in A. c. cerana workers' midguts responding to $N$. ceranae challenge.

- This data will enable a better understanding of the molecular mechanism regulating eastern honeybee- $N$. ceranae interaction.

\section{Data}

The shared datasets were derived from strand-specific cDNA library-based RNA-seq of un-infected (AcCK1 and AcCK2) and N. ceranae-infected (AcT1 and AcT2) A. c. cerana workers' midguts [1]. Totally, 56270223688, 44860946964, 78991623806, and 92712308296 raw reads were gained from AcCK1, AcCK2, AcT1 and AcT2, respectively (Table 1). In addition, 54495191388, 43570608753, 76708161525 , and 89467858351 clean reads were obtained after strict quality control, with Q30 value of 95.80\%, 95.99\%, 96.07\%, and 96.04\%, and GC content of 44.20\%, $43.44 \%, 44.83 \%$, and $43.63 \%$, respectively (Table 1). Pearson correlation coefficients among three replicas in each group were above 0.8772 (Fig. 1). The raw data were submitted to the NCBI Sequence Read Archive (SRA) database and connected to BioProject PRJNA562784.

\section{Experimental Design, Materials, and Methods}

\subsection{N. ceranae spore purification}

$N$. ceranae spores were previously purified from midguts of Apis mellefera ligustica workers infected by N. ceranae [2]. The spore suspension was freshly prepared before use.

\subsection{Experimental design and sample collection}


Frames of sealed brood obtained from a healthy colony of A. c. cerana located in the teaching apiary of College of Animal Sciences (College of Bee Science), Fujian Agriculture and Forestry University were reared in an incubator at $34 \pm 0.5{ }^{\circ} \mathrm{C}, 50 \%$ RH to provide newly emerged Nosema-free honeybees. The emergent workers were carefully removed and confined to cages in groups $(n=20)$, and kept in the incubator at $32 \pm 0.5^{\circ} \mathrm{C}, 50 \% \mathrm{RH}$.

The workers were fed ad libitum with a f sucrose solution (50\% w/v in water), and one day after eclosion, workers in treatment groups were starved for $2 \mathrm{~h}$ and 20 workers per group were each immobilized and then fed with $5 \mathrm{uL}$ of $50 \%$ sucrose solution containing $1 \times 10^{6}$ fungal spores. Those workers that did not consume the total amount of sucrose solution were discarded. After inoculation, workers were isolated for $30 \mathrm{~min}$ in vials in the growth chamber to ensure that the sucrose solution was not transferred among individuals and the entire dosage was ingested. Workers in control groups were inoculated in an identical manner using a 50\% sucrose solution (w/w in water) without $N$. ceranae spores. Three replicate cages of 20 honeybees each were used in treatment and control groups. Each cage was checked every $24 \mathrm{~h}$ and any dead bees removed. N. ceranae-infected and un-infected workers' midguts were respectively harvested at $7 \mathrm{~d}$ or $10 \mathrm{~d}$ post inoculation (dpi), immediately frozen in liquid nitrogen and kept at $-80{ }^{\circ} \mathrm{C}$ until RNA sequencing. $N$. ceranae-infected groups at $7 \mathrm{dpi}$ and $10 \mathrm{dpi}$ with sucrose solution containing $N$. ceranae spores were termed as AcT1 (AcT1-1, AcT1-2, AcT1-3) and AcT2 (AcT2-1, AcT2-2, AcT2-3); un- infected groups at $7 \mathrm{dpi}$ and $10 \mathrm{dpi}$ with sucrose solution without $N$. ceranae spores were termed as AcCK1 (AcCK1-1, AcCK1-2, AcCK1-3) and AcCK2 (AcCK2-1, AcCK2-2, AcCK2-3).

\subsection{Strand-specific cDNA library construction and illumina sequencing}

Firstly, total RNA of the six midgut samples from N. ceranae-infected groups and six midgut samples from un-infected groups were respectively extracted using Trizol (Life Technologies) following the manufacturer's protocol, and examined via $1 \%$ agarose gel eletrophoresis. Secondly, rRNAs were removed to retain mRNAs and non-coding 
RNAs (ncRNAs), which were fragmented into short fragments with fragmentation buffer (Illumina, USA) followed by reverse transcription into cDNA with random primers. Thirdly, second-strand cDNA were synthesized by dNTP (dUTP instead of dTTP), DNA polymerase I, RNase H, and buffer. Fourthly, the cDNA fragments were purified using QiaQuick PCR extraction kit (QIAGEN, Germany), end repaired, poly(A) added, and ligated to Illumina sequencing adapters, followed by digestion of the second-strand cDNA with UNG (Uracil-N-Glycosylase) (Illumina, USA) . Finally, the digested products were size selected by agarose gel electrophoresis, PCR amplified, and sequenced on Illumina HiSeq ${ }^{\mathrm{TM}} 4000$ platform (Illumina, USA) by Gene Denovo Biotechnology Co. (China). The raw sequencing data produced in this article are available in NCBI SRA database and connected to BioProject PRJNA562784.

\subsection{Quality control of raw reads and mapping of clean reads}

Firstly, raw reads were filtered by removing reads containing adapters, more than $10 \%$ of unknown nucleotides $(\mathrm{N})$, and more than $50 \%$ of low quality bases to obtain high quality clean reads. Quality control of transcriptome data is showed in Table 1. Secondly, the filtered raw reads were mapped to ribosome RNA (rRNA) database using short reads alignment tool Bowtie2 [3] . Thirdly, the mapped reads were removed and the remaining reads were used in assembly and further analysis. The rRNA-removed reads of each sample were then mapped to Apis cerana genome (assembly ACSNU-2.0) using TopHat2 (version 2.0.3.12) [4] following alignment parameters: (1) maximum read mismatch is two; (2) the distance between mate-pair reads is $50 \mathrm{bp}$; (3) the error of distance between mate-pair reads is $\pm 80 \mathrm{bp}$.

\subsection{Transcript assembly}

Transcripts were assembled using Cufflinks [5]. The program reference annotation based transcripts (RABT) was preferred. Cufflinks constructed faux reads based on reference to make up for the influence of low coverage sequencing. During the last step of assembly, all of the reassembles fragments were aligned with reference genes followed by removel of similar fragments. Cuffmerge was used to merge transcripts from different replicas of a group into a comprehensive set of transcripts, and the transcripts from multiple groups were then merged into a final set of transcripts. 
Pearson correlation coefficients between every biological replicas in each sample group were calculated and presented in Fig. 1.

\section{Acknowledgments}

This work was supported by the Earmarked Fund for Modern Agro-industry Technology Research System (CARS-44-KXJ7), the Science and Technology Planning Project of Fujian Province (2018J05042), the Education and Scientific Research Program Fujian Ministry of Education for Young Teachers (JAT170158), the Outstanding Young Scientific Research Talents Program of Fujian Agriculture and Forestry university (xjq201814), and the Fujian Agriculture and Forestry University Science and Technology Innovation Special Fund (CXZX2017342, CXZX2017343).

\section{Reference}

[1] Z. M. Fu, D.D. Zhou, H.Z. Chen, S.H. Geng, Y.Z. Zheng, C.L. Xiong, G.J. Xu, $\mathrm{X}$. Zhang, R. Guo. Analysis of highly expressed genes in Apis cerana cerana workers' midguts responding to Nosema ceranae stress. J. Sichuan Univ. (Nat. Sci. Ed.) 57(2020) 191.

[2] H.Z. Chen,Y. Du, C.L. Xiong, Y.Z. Zheng, D.F. Chen, R. Guo. A comprehensive transcriptome data of normal and Nosema ceranae -stressed midguts of Apis mellifera ligustica workers. Data in Brief (2019) 26.

[3] B. Langmead, S.L. Salzberg, Fast gapped-read alignment with Bowtie 2, Nat. Methods 9 (2012) 357e359.

[4] D. Kim, G. Pertea, C. Trapnell, H. Pimentel, R. Kelley, S.L. Salzberg, TopHat2: accurate alignment of transcriptomes in the presence of insertions, deletions and gene fusions, Genome Biol. 14 (2013) R36.

[5] C. Trapnell, A. Roberts, L. Goff, G. Pertea, D. Kim, D.R. Kelley, H. Pimentel, S.L. Salzberg, J.L. Rinn, L. Pachter, Differential gene and transcript expression analysis of RNA-seq experiments with TopHat and Cufflinks, Nat. Protoc. 7 (2012) 562e578. 
Table 1 Overview of strand-specific cDNA library-based RNA-seq data

\begin{tabular}{lllll}
\hline Samples & Raw reads (bp) & Clean reads (bp) & $Q 30$ & GC content \\
\hline AcCK1-1 & 16885642346 & 16399161584 & $95.87 \%$ & $45.48 \%$ \\
AcCK1-2 & 17755250138 & 17263781337 & $96.00 \%$ & $44.35 \%$ \\
AcCK1-3 & 21629331204 & 20832248467 & $95.54 \%$ & $42.76 \%$ \\
AcCK2-1 & 16737021804 & 16268561216 & $96.11 \%$ & $42.21 \%$ \\
AcCK2-2 & 13163497480 & 12763936876 & $95.85 \%$ & $45.17 \%$ \\
AcCK2-3 & 14960427680 & 14538110661 & $96.02 \%$ & $42.93 \%$ \\
AcT1-1 & 23052018714 & 22411065486 & $96.20 \%$ & $42.64 \%$ \\
AcT1-2 & 23219116354 & 22562514467 & $96.13 \%$ & $44.54 \%$ \\
AcT1-3 & 32720488738 & 31734581572 & $95.87 \%$ & $47.30 \%$ \\
AcT2-1 & 30973453890 & 29309979248 & $95.78 \%$ & $45.96 \%$ \\
AcT2-2 & 39850701244 & 38878740450 & $96.29 \%$ & $41.96 \%$ \\
AcT2-3 & 21888153162 & 21279138653 & $96.04 \%$ & $42.98 \%$ \\
\hline
\end{tabular}



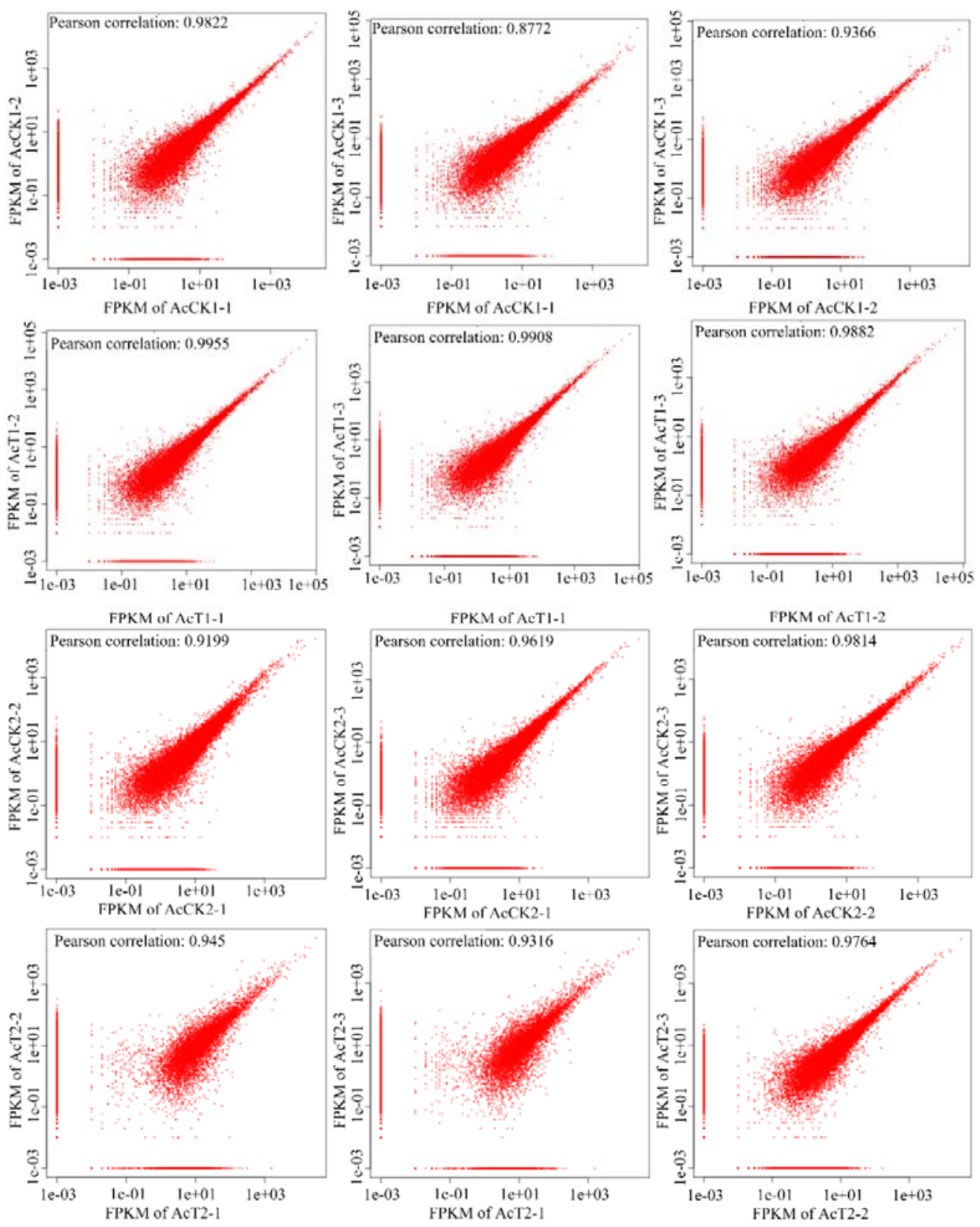

Fig. 1. Pearson correlation coefficients among different replicas within every un-infected and N. ceranae-infected groups. 